\title{
The Effect of Wood on the Sound Quality of Electric String Instruments
}

\author{
J. Puszyński*, W. Moliński And A. PReis \\ Department of Wood Science, Poznań University of Life Sciences, Wojska Polskiego 38/42, 60-627 Poznań, Poland \\ and \\ Institute of Acoustics, Adam Mickiewicz University, Umultowska 85, 61-614 Poznań, Poland
}

\begin{abstract}
The objective of the study was to investigate the influence of wood species on sound timbre generated by electric string instruments. The obtained results allow to conclude that wood species have the largest influence on the specific sound loudness and it correlates with density and modulus of elasticity. The greater differences occur at lower frequencies. There was no correlation between signal recorded by microphone and pickup.
\end{abstract}

DOI: $10.12693 /$ APhysPolA.127.114

PACS: $43.75 .+\mathrm{a}, 83.80 . \mathrm{Mc}$

\section{Introduction}

Acoustic properties of wood have been thoroughly investigated in many researches. Resonance wood should meet specific requirements imposed in manufacture of musical instruments such as high quality, defect-free wood with narrow growth rings, low density and potentially the highest modulus of elasticity (MOE) [1-4]. However, the vast majority of that knowledge is dedicated to acoustical instruments and their soundboards (mainly violins).

The function and effect of wood on electric string instruments are poorly understood. Most controversies are connected with instruments' sound timbre. Some musicians and producers believe that the wood species used to build that group of instruments affects sound timbre, while others are of an opposite opinion. On the other hand, some wood species are more willingly and often used than others. This group of instruments is characterized by a great variety of wood species used in their production. Of course, the choice of wood depends on many others factors e.g. material durability, dimensional stability, mass and esthetical values. Moreover, many controversies might occur due to subjective judgments of sound timbre. What is more, sound quality of electric string instruments depends on strings, amplifiers, speakers, special sound effects, concerts halls or recording rooms, etc. To evaluate subjective sound perception psychoacoustics parameters should be used such as e.g. sharpness, roughness and specific loudness [5]. In most cases when a sound signal is generated in the case of these instruments the only source is electromagnetic pickup. Then the generated signal depends only on metal string vibrations $[6-10]$.

The aim of this study was to examine how wood species, its density and MOE affect sound timbre in that group of instruments.

${ }^{*}$ corresponding author; e-mail: puszynski@gmail.com

\section{Method}

To simplify the complexity of design a model replaced the actual guitar. Each model consists of a plank of selected wood species with constant dimensions, a tuner, a bridge and an attached string (Fig. 1). The length of the string scale is similar to the one in standard electric guitars. Four wood species were used: maple [Acer pseudoplatanus L.], ash [Fraxinus excelsior L.], alder [Alnus glutinosa L.] and cedar [Cedrus sp.]. Maple, ash and alder are popular species in guitar building. Cedar is an exception. It was chosen due to its very low density and MOE. Four planks were made from each species.
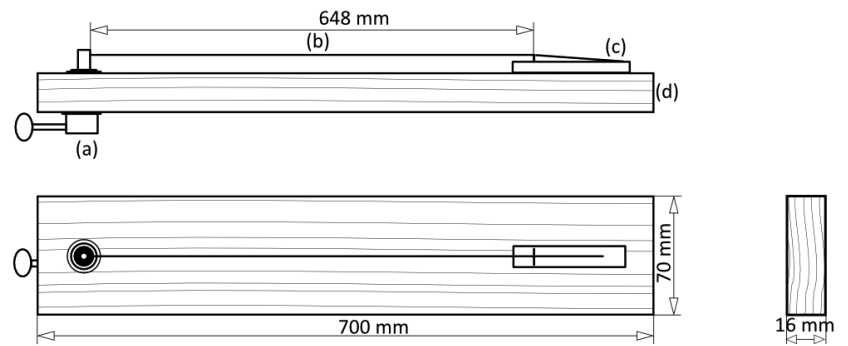

Fig. 1. Models with mounted bridge (c), tuner (a) and attached string (b) on the wooden base. We can see also how wood rings were oriented.

Before final cutting, wood was specifically prepared. It was obtained from the Mabor sawmill (Swarzędz, Poland). Air-dried, unedged timber after 3 month seasoning in the sawmill was delivered to the laboratory. From each timber sample one plank with overages was worked and conditioned for the next three months (38 \pm $2 \%$ relative humidity, $17.2 \pm 1{ }^{\circ} \mathrm{C}$ temperature). After that, planks were cut to final dimensions. All planks were oriented in the same, longitudinal direction. In the cross-section, the tangential direction was parallel to the longer axis. Moisture content of all planks was equilibrium moisture content to ambient conditions and it was similar for each wood species $(8 \%)$. In the meantime wood properties were measured. Sample mass $( \pm 0.01 \mathrm{~g})$, 
divided by three dimensions of regularly shaped planks $( \pm 0.05 \mathrm{~mm})$, i.e. its volume gives wood density. Sound velocity in the longitudinal direction was measured on the Clpan material tester type 543E. Dynamic MOE was calculated based on sound velocity in the longitudinal direction and density. A special test stand was designed to put string into vibrations. Models are always put on acoustic foam in the same position. A pendulum with a mounted guitar pick was used to provide string vibrations with constant energy. Pick position, relative to the string, was continuously monitored.

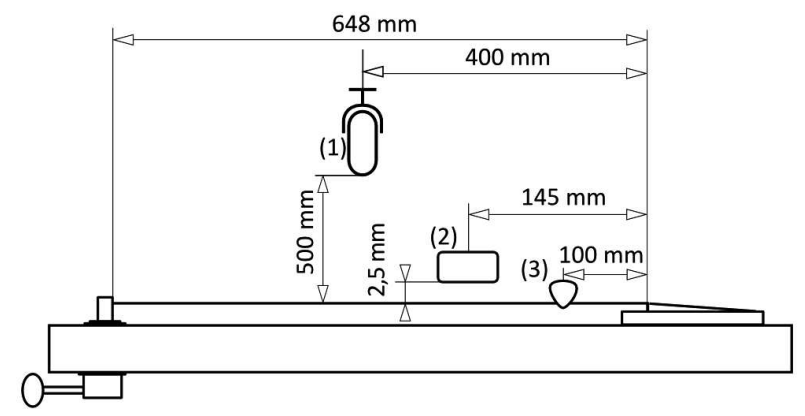

Fig. 2. Test stand scheme with: microphone (1), electromagnetic pickup (2) and guitar pick (3) mounted on the pendulum.

At the same time, sound was recorded with a pickup and microphone (Fig. 2): an electromagnetic, active pickup type humbucker: Bartolini E 94-D Active, powered by a $9 \mathrm{~V}$ battery and a GARS 40 AN microphone with a SVAN SV 08A preamplifier. The signal was recorded via a USB interface Saffire PRO 26 on the Cubase software. All records were carried out in an anechoic chamber. There were five different strings recorded on each model: E6-B2 with a $83-247 \mathrm{~Hz}$ frequency range. Each configuration was recorded five times. Recorded sound samples were analysed on Artemis software. The analysed psychoacoustic parameters included roughness [asper], sharpness [acum] and specific loudness [sone].

\section{Results and discussion}

Wood used in this study was characterized by highly variable density and MOE. Wood density ranged from 310 to $691 \mathrm{~kg} / \mathrm{m}^{3}$. MOE in the longitudinal direction ranged from 7097 to $19653 \mathrm{MPa}$. These data provide a broad spectrum of wood properties used for the test. Moreover, the results confirm the known fact of the linear relationship between wood density and MOE (Fig. 3).

Roughness measured for microphone signal for particular wood species and string shows that the effect of strings on roughness is much more marked than that of wood (Fig. 4). This effect is ambiguous, i.e. different for each string. For e6 string $(83 \mathrm{~Hz})$ roughness ranged from 1.48 to 1.70 [asper] (alder and maple) with standard deviation \pm 0.06 . b2 string roughness $(247 \mathrm{~Hz})$ ranged from 0.37 (maple) to 0.49 [asper] (cedar) with \pm 0.07 average

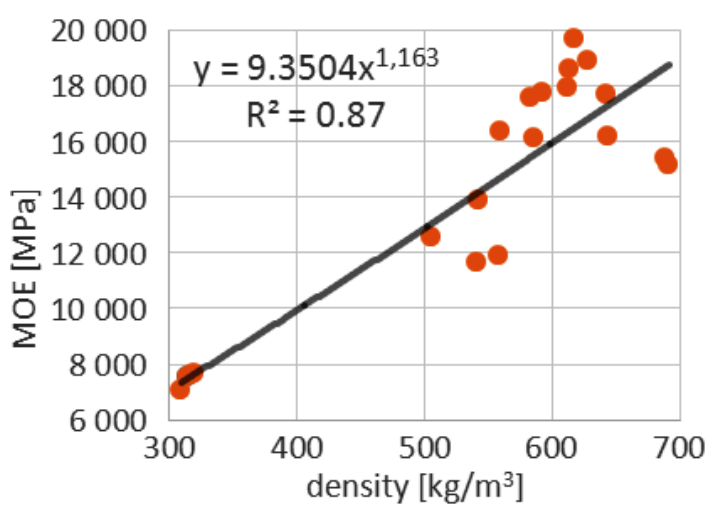

Fig. 3. Relation between wood density and modulus of elasticity.

standard deviation. We can see that a lower frequency gives greater roughness variations of tested wood species with a smaller standard deviation.

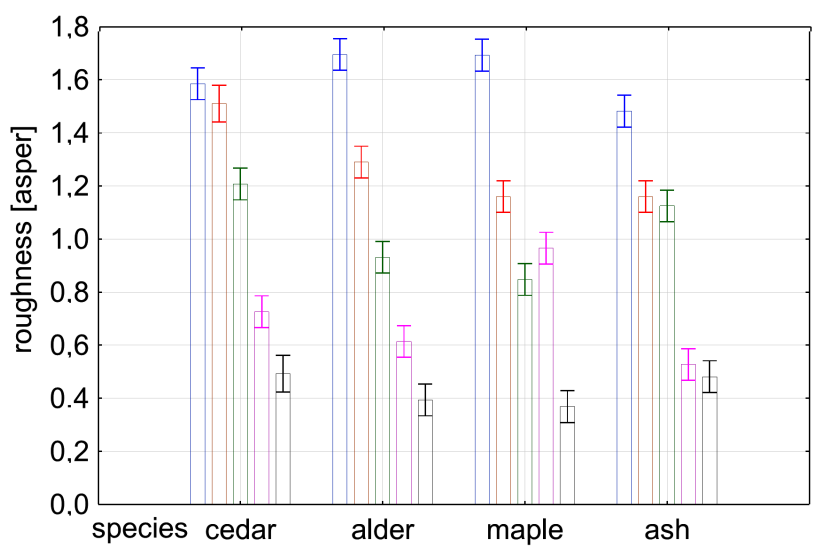

Fig. 4. Comparison of values of roughness measured for signal recorded by microphone for different wood species and for each string (from the left: e6, a5, $\mathrm{d} 4, \mathrm{~g} 3, \mathrm{~b} 2)$.

Roughness measured for electromagnetic pickup signal does not depend so much on used wood species (Fig. 5). The range of roughness values for each string recorded via a pickup (0.3-2.5 asper) is much greater than the recorded microphone signal (1.7-0.37 asper). A lesser effect of wood species on roughness also provides no correlation of this value with density or MOE.

Results of sharpness measured for signal recorded with a microphone and a pickup are similar to those for roughness. A thorough analysis of these results showed that the effect of wood species is ambiguous and less marked than that of roughness. In addition, statistical analysis of roughness and sharpness results using the Tukey test indicated that wood species does not affect significantly ( $p=0.9-0.1)$ analysed volume.

Specific loudness measured for the microphone signal has an interesting effect depending on wood 


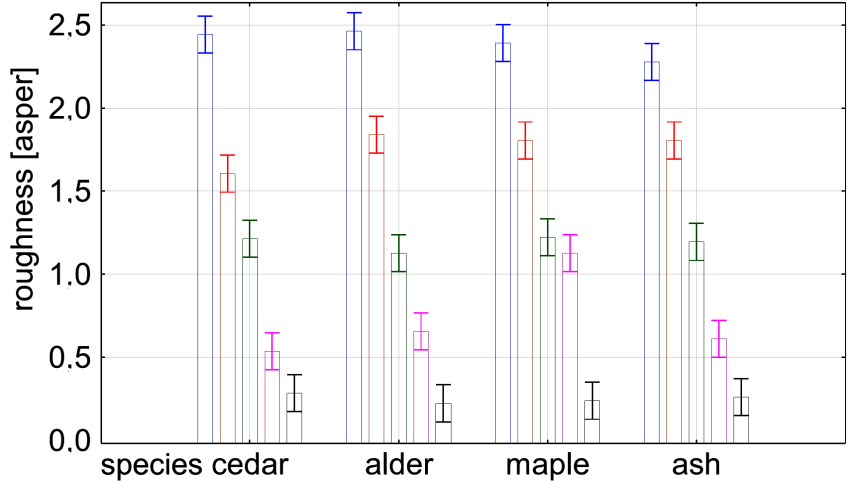

Fig. 5. Comparison of values of roughness measured for signal recorded electromagnetic pickup for different wood species and for each string (from the left: e6, a5, $\mathrm{d} 4, \mathrm{~g} 3, \mathrm{~b} 2)$.

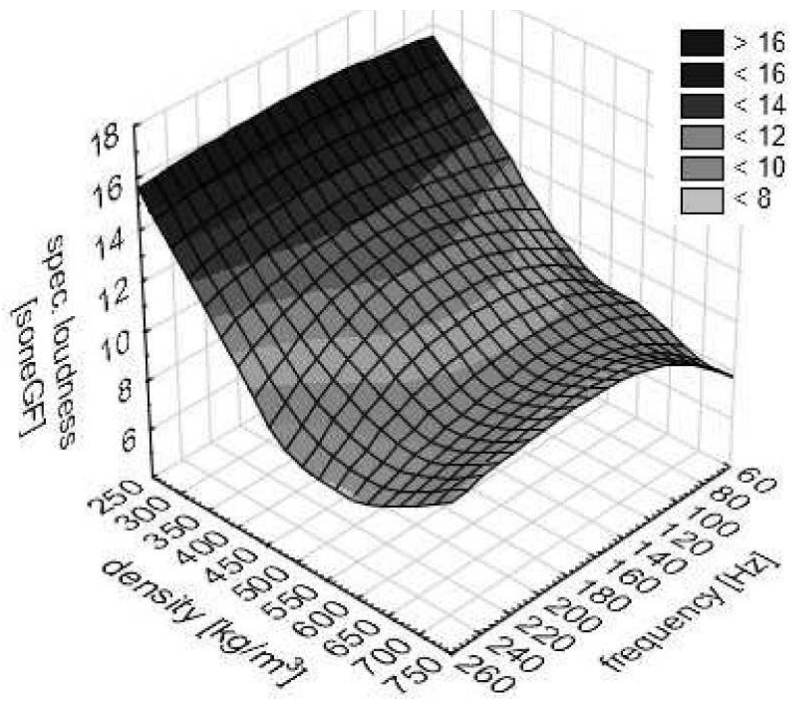

Fig. 6. Relation between density, frequency and specific loudness measured for microphone signal.

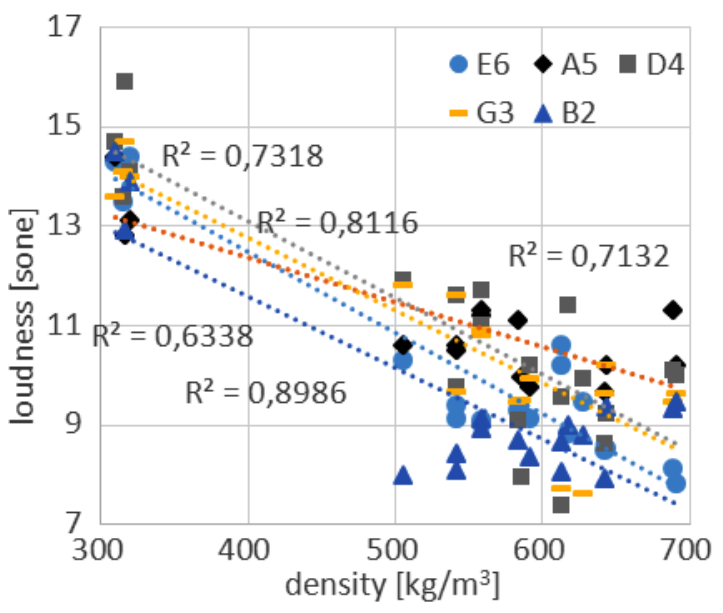

Fig. 7. Relation between density and specific loudness measured for microphone signal. species (Fig. 6). Specific loudness ranged from 8.8 to 14.4 [soneGF] for e6 string and from 6.31 to 13.9 [soneGF] for string b2. Between previously tested species of wood, cedar had the highest roughness value and ash and maple - the lowest. Minimum and maximum specific loudness values were 2 -fold greater. Figure 7 shows that specific loudness is negatively correlated with wood density $\left(R^{2}=0.63-0.90\right)$ - and referring to the relationship shown in Fig. 3 - that correlation also applies to MOE $\left(R^{2}=0.57-0.70\right)$. This fact can be explained by a higher vibration susceptibility to materials with lower inertia (mass).

\section{Conclusions}

Based on the results, it can be concluded that:

1. Wood species has an ambiguous effect on sound timbre of recorded signals and it depends on the recording method (microphone, pickup).

2. Statistical analysis of roughness and sharpness results showed a non-significant, ambiguous effect of wood species on these parameters.

3. Wood species affects specific loudness for signal recorded using a microphone. In that case, correlations between wood density (also MOE) and specific loudness are linear $(r=-0.7$ to -0.95$)$. Pickup signals give no correlations.

\section{References}

[1] V. Bucur, Acoustics of Wood. Springer Series in Wood Science, 2nd ed., Springer-Verlag, Berlin 2005.

[2] C. Buksnowitz, A. Teischinger, J. Acoust. Soc. Am. 121, 2384 (2007).

[3] T. Ono, M. Norimoto, Rheol. Acta 23, 652 (1984).

[4] U.G.K. Wegst, Am. J. Botany 93, 1439 (2006).

[5] H. Fastl, E. Zwicker, Psychoacoustics - Facts and Models. Springer series, Springer, Berlin 2007.

[6] T. Fanuel Ban, Analysis of Electric Guitar Pickups, LMS North. America, Huntington Beach (CA) 2010.

[7] H. Fleischer, Dead Spots of Electric Guitars and Basses, popular version of paper 5aMUb6, 1999.

[8] J. Lähdevaara, The Science of Electric Guitars and Guitar Electronics, 2014.

[9] J. Puszyński, "String-wood feedback in electric string instruments", Ann. WULS-SGGW Forestry and Wood Technology, in press, 2014.

[10] D.T. Rossing, The Science of String Instruments, Center for Computer Research in Music and Acoustics (CCRMA), Stanford (CA) 2010. 